\title{
Homogenization and error estimates of free boundary velocities in periodic media
}

\author{
Inwon C. Kim
}

October 7, 2011

\begin{abstract}
In this note I describe a recent result ([14]-[15]) on homogenization and error estimates of a free boundary problem, which describes quasi-static contact angle dynamics on inhomogeneous surface. The method presented here also applies to more general class of free boundary problems with oscillating boundary velocities.
\end{abstract}

Let us define $e_{i} \in \mathbb{R}^{n}, i=1, \ldots, n$ such that

$$
e_{1}=(1,0, . ., 0), e_{2}=(0,1,0, . ., 0), \ldots, \text { and } e_{n}=(0, \ldots, 0,1),
$$

and consider a Lipschitz continuous function

$$
g: \mathbb{R}^{n} \rightarrow[m, M], g\left(x+e_{i}\right)=g(x) \text { for } i=1, \ldots, n
$$

with Lipschitz constant $L$. In this paper we are interested in the behavior, as $\epsilon \rightarrow 0$, of the viscosity solutions $u^{\epsilon} \geq 0$ of the following problem with $K=\{|x| \leq 1\}$ and with initial data $u_{0}$ :

$(P)_{\epsilon}$

$$
\begin{cases}-\Delta_{x} u^{\epsilon}(\cdot, t)=0 & \text { in } \quad\left\{u^{\epsilon}>0\right\}-K \\ u_{t}^{\epsilon}=\left|D_{x} u^{\epsilon}\right|\left(\left|D_{x} u^{\epsilon}\right|-g\left(\frac{x}{\epsilon}\right)\right) & \text { on } \quad \partial\left\{u^{\epsilon}>0\right\} \\ u=1 & \text { on } \quad K .\end{cases}
$$

We refer to $\Gamma_{t}\left(u^{\epsilon}\right):=\partial\left\{u^{\epsilon}(\cdot, t)>0\right\}-\partial K$ as the free boundary of $u^{\epsilon}$ at time $t$. Note that if the free boundary of $u^{\epsilon}$ is smooth, then the boundary moves with outward normal velocity $V=\frac{u_{t}^{\epsilon}}{\left|D u^{\epsilon}\right|}$, and therefore the second equation in $(P)_{\epsilon}$ implies that

$$
V=\left|D u^{\epsilon}\right|-g\left(\frac{x}{\epsilon}\right)=D u^{\epsilon} \cdot(-\nu)-g\left(\frac{x}{\epsilon}\right)
$$

where $\nu=\nu_{x, t}$ denotes the outward normal vector at $x \in \Gamma_{t}(u)$ with respect to $\Omega_{t}(u)$.

$(P)_{\epsilon}$ is a simplified model to describe contact line dynamics of quasi-static liquid droplets on an irregular surface $([11])$. Here $u(x, t)$ denotes the height of the droplet. Heterogeneities on the surface, represented by $g\left(\frac{x}{\epsilon}\right)$, result in contact lines with a fine scale structure that may lead to pinning of the interface and hysteresis of the overall fluid shape.

The following is a summary of the main result in [14]: 
Theorem 1.1. Let $u^{\epsilon}$ be a viscosity solution of $(P)_{\epsilon}$ with initial data $u_{0}$. Then there exists a continuous function $r(q)=\mathbb{R}^{n}-\{0\} \rightarrow[-2, \infty)$, increasing with respect to $|q|$, such that the following holds: Let $(P)$ be the free boundary problem derived by replacing the second condition in $(P)_{\epsilon}$ by $u_{t}=|D u| r(D u)$. If $u$ is the unique viscosity solution of $(P)$ with initial data $u_{0}$, then $u_{\epsilon}$ locally uniformly converges to $u$.

A brief outline of the proof for above theorem is laid out in section 3. There is an extensive amount of literature on the subject of homogenization. For detailed survey on different approaches we refer to [5]. For free boundary problems, very little is known due to the difficulties arising from the lowerdimensional nature of the interface: for example the periodicity of $g$ in $(P)_{\epsilon}$ does not guarantee the interface $\Gamma_{t}\left(u_{\epsilon}\right)$ to be periodic in space. Due to the lack of variational structure for $(P)_{\epsilon}$, it seems difficult - and perhaps not possible - to get an explicit expression of $r(q)$. On the other hand some explicit results are available for problems with variational structure ([6], [16]-[17]). Also see results for traveling waves for the flame propagation([2]-[3]), which make use of the phase-field approximation.

In [13]-[15] we follow the perturbed test function method of [10] and the obstacle approach of [5] to study free boundary problems with oscillating velocity. Roughly speaking, the limiting velocity law is established by finding the "corrector" $P_{q, r}$ in the following sense: for given $q \in \mathbb{R}^{n}-\{0\}$, show that there is a unique speed $r=r(q)$ such that the $u^{\epsilon}$ satisfying in $Q_{1}:=B_{1}(0) \times[0,1]$

$$
\left\{\begin{array}{l}
-\Delta u^{\epsilon}=0 \text { in }\left\{u^{\epsilon}>0\right\} ; \quad V=\left|D u^{\epsilon}\right|-g\left(\frac{x}{\epsilon}\right) \text { on } \partial\left\{u^{\epsilon}>0\right\} ; \\
u^{\epsilon}=P_{q, r}(x, t):=|q|\left(r t-x \cdot \frac{q}{|q|}\right)_{+} \quad \text { on the parabolic boundary of } Q_{1} .
\end{array}\right.
$$

stays close to $P_{q, r}$. In [15] quantitative estimates on the distance between $u^{\epsilon}$ and $P_{q, r}$ for different speeds $r$ are obtained to derive the following error estimate:

$$
\text { For sufficiently small } \epsilon>0, \Omega_{t}\left(u^{\epsilon}\right) \text { stays in } O\left(\epsilon^{1 / 70}\right) \text {-neighborhood of } \Omega_{t}(u) \text { for } 0 \leq t \leq \epsilon^{-1 / 100} \text {. }
$$

Let us mention that $r(q)$ is in general dependent on both $|q|$ and the direction of the interface $\frac{q}{|q|}$, and $r(q)$ may be zero for a positive measure of $q$ (see section 4, [14]). Furthermore for general $g$, preliminary analysis ([7]) yields that for any fixed $q \in \mathbb{R}^{n}$, if $a_{0}$ is the biggest constant for which $r(a q)=0$, then $r\left(\left(a+a_{0}\right) q\right) \sim a^{1 / 2}$ for $a>0$. The effect of the structure of different $g$ 's on $r(q)$ is an interesting, and challenging open question.

This note is organized as follows: In section 2 I will introduce important properties of viscosity solutions that are used in the paper. In section 3 the proof of Theorem 1.1 is outlined. In section 4 we state and discuss the proof of the error estimates. Since the goal here is to introduce the key ideas, I will not state all the necessary assumptions and will not describe all the details.

It should be noted that the method outlined in this note makes strong use of periodicity of $g$ in space, and thus it does not directly extend to the case of random media. On the other hand, our method extends to a general class of free boundary problems which satisfy the comparison principle (see $[15])$. 


\section{Comparison principle and Sup-/Inf-convolutions}

We use the notion of viscosity solutions to describe the solution of $(P)_{\epsilon}$ and $(P)$. The notion of weak notions are necessary since, even with smooth initial data, one cannot expect classical solutions to exist in global time. In fact solutions may develop singularities in finite time due to collision or pinch-off of different free boundary parts.

Viscosity solutions were originally introduced by Crandall and Lions (see e.g., [8], [9]) for HamiltonJacobi equations and has been successfully extended to various nonlinear PDEs and free boundary problems. These solutions are defined with basis on maximum principle-type arguments and are rather stable with respect to topological changes of interfaces and singularity formations. We refer to [15] for the definition of viscosity solutions in our setting: here we will state the comparison principle which is essential in the analysis presented in our note.

We say that a pair of functions $u_{0}, v_{0}: \bar{D} \rightarrow[0, \infty)$ satisfy $u \prec v$ in $D \subset \mathbb{R}^{n}$ if $u_{0}(x)<v_{0}(x)$ in the support of $u_{0}$ in $\bar{D}$.

Theorem 2.1. [Comparison Principle, [14]] Let $F(p, x): \mathbb{R}^{n}-\{0\} \times \mathbb{R}^{n} \rightarrow \mathbb{R}$ be continuous with respect to $x, p$ and increasing with respect to $|p|$. Let $u$ and $v$ be respectively a viscosity sub- and supersolution of the following generalized problem

$$
\left\{\begin{array}{l}
-\Delta w(\cdot, t)=0 \text { in }\{w>0\} \\
w_{t}=|D w| F(D u, x)
\end{array}\right.
$$

in a cylindrical domain $\Sigma=\mathcal{B} \times[a, b] \subset \mathbb{R}^{n} \times \mathbb{R}$. If $u \prec v$ in the parabolic boundary of $\Sigma$, then $u \prec v$.

Based on the comparison principle, we have existence and uniqueness for viscosity solutions of the limit problem $(P)$ if the initial data satisfies one of the following (see [15]):

(I-a) $\Omega$ is star-shaped with respect to a small ball $B_{r}(0)$;

(I-b) $\Gamma_{0}:=\partial\left\{u_{0}>0\right\}$ is locally Lipschitz and $\left|D u_{0}\right|>2$ on $\Gamma_{0}$;

(I-c) $\Gamma_{0}$ is locally Lipschitz and $\left|D u_{0}\right|<1$ on $\Gamma_{0}$.

For free boundary problems, the following Sup- and Inf-convolutions are frequently used (e.g. see section 4 of this note) to generate regularized perturbations of solutions:

Lemma 2.2 (Lemma 3.5, [12]). (a) If u is a viscosity subsolution of $(P)$, then the sup-convolution of $u$

$$
\tilde{u}(x, t):=\sup _{|x-y| \leq m \epsilon-\delta t} u(y, t)
$$

is a viscosity subsolution of $(P)$ for $0 \leq t \leq \frac{m \epsilon}{\delta}$, with $r(D u)$ replaced by $r(D u)-\delta$.

(b) If $u$ is a supersolution of $(P)$, then the inf-convolution of $u$

$$
\bar{u}(x, t):=\inf _{|x-y| \leq m \epsilon-\delta t} u(y, t)
$$

is a viscosity supersolution of $(P)$ with $r(D u)$ replaced by $r(D u)+\delta$. 
The regularity properties of the convoluted function $\tilde{u}$ and $\bar{u}$ are as follows: for example, any point $x_{0} \in \Gamma_{t}(\tilde{u})$, there will be a spatial ball $B\left(y_{0}\right)$ of radius $m \epsilon-\delta t$ which lies in the positive set $\{\tilde{u}(\cdot, t)>0\}$ and touches $x_{0}$ on its boundary. In other words $\Gamma(\tilde{u})$ is " $C^{2}$ from inside" in space. On the other hand, $\Gamma(\bar{u})$ is " $C^{2}$ from outside" in space variables. These half-regularities often comes in handy with maximum-principle type arguments: see the discussion in section 4.

One can also use the space-time balls instead of the space balls used in the definition of $\tilde{u}$ and $\bar{u}$ to obtain space-time regularity properties of the free boundary.

\section{Obstacle problem and uniqueness of the limiting slope}

In this section we introduce the obstacle problems, and describe their contribution to the proof of Theorem 1.1. To define the limiting free boundary velocity we apply the ideas in [5], which studies homogenization limits of fully nonlinear equations in ergodic random media. The main idea in the analysis of [5] is that, to describe the limiting problem, it is enough to decide whether a given test function is either a subsolution or a supersolutions of the problem. The test functions were quadratic polynomials in [5] since the equation under investigation was of second-order, but for our problem, whose motion law is of first order, the corresponding test functions are the traveling wave solutions,

$$
P_{q, r}(x, t):=|q|(r t-x \cdot \nu)_{+}, \nu=\frac{q}{|q|}
$$

whose free boundary, which is a hyperplane, propagates with given speed $r \in \mathbb{R}$ and normal direction $q \in \mathbb{R}^{n}$ of the propagation (see section 2). Let us denote the free boundary of $P_{q, r}$ at time $t$ by $l_{q, r}(t):=\left\{x \in \mathbb{R}^{n}: r t=x \cdot \nu\right\}$.

Next, for a ball-like domain $\mathcal{D} \subset \mathbb{R}^{n}$ containing the origin ( see section 3 of [15] for detailed description of $\mathcal{D}$ ), we consider the maximal subsolution below and the minimal supersolution above $P_{q, r}$ in the cylindrical domain $Q_{1}=\mathcal{D} \times[0,1]$ :

$$
\begin{gathered}
\bar{u}_{\epsilon ; q, r}:=\sup \left\{u: \text { a viscosity subsolution of }\left(P_{\epsilon}\right) \text { in } Q_{1} \text { with } u \leq P_{q, r}\right\}, \\
\underline{u}_{\epsilon ; q, r}:=\inf \left\{v: \text { a viscosity supersolution of }\left(P_{\epsilon}\right) \text { in } Q_{1} \text { with } u \geq P_{q, r}\right\} .
\end{gathered}
$$

Note that if $r$ is big then the free boundary of $\bar{u}$ will lag behind $l_{q, r}$ in the interior of $Q_{1}$, and if $r$ is small then the free boundary of $\underline{u}$ will advance away from $l_{q, r}$. The main result in section 3 of [15] can be summarized as follows:

Proposition 3.1. For each $q \in \mathbb{R}^{n}$ there exists a unique $r=r(q)$ such that both the contact sets

$$
\bar{A}_{\epsilon ; q, r}:=\Gamma\left(\bar{u}_{\epsilon ; q, r}\right) \cap l_{q, r} \text { and } \underline{A}_{\epsilon ; q, r}:=\Gamma\left(\underline{u}_{\epsilon ; q, r}\right) \cap l_{q, r}
$$

are nonempty in

$$
\tilde{Q}_{1}:=\{x: d(x, \partial \mathcal{D})>1 / 10\} \times[1 / 2,1]
$$

for all $\epsilon>0$.

We sketch the proof of above proposition below.

First let us point out that there are monotonicity properties of the contact sets, which makes the analysis easier for the obstacle solutions $\bar{u}$ and $\underline{u}$ than for solutions without obstacles. It is easy 
to see from the free boundary velocity that the contact set $\bar{A}_{\epsilon ; q, r}$ increases as $r$ decreases and $|q|$ increases, and $\underline{A}_{\epsilon ; q, r}$ decreases as $r$ increases and $|q|$ decreases. Moreover, a scaling argument yields that decreasing $\epsilon$ in the definition of $\bar{u}_{\epsilon}$ or $\underline{u}_{\epsilon}$ is equivalent to fixing $\epsilon$ and making the domain $Q_{1}$ larger. Using this fact one can show certain monotonicity properties between obstacle solutions for different $\epsilon$ 's: for example show that if $\bar{A}_{\epsilon ; q, r}$ becomes empty for $\epsilon_{0}$, then it would be nonempty for all $\epsilon<\epsilon_{0}$.

Let us define

$$
\bar{r}(q):=\sup \left\{r: \bar{A}_{\epsilon ; q, r} \text { is nonempty for sufficiently small } \epsilon>0\right\}
$$

and

$$
\underline{r}(q):=\inf \left\{r: \underline{A}_{\epsilon ; q, r} \text { is nonempty for sufficiently small } \epsilon>0\right\} .
$$

Our goal in this section is to give a brief summary of section 3 in [14], which proves that $\bar{r}(q)=$ $\underline{r}(q)$. This unique speed then will be our candidate for the homogenized normal velocity $r(q)$. The perturbed test function method of [10] is tailored so that to get homogenization it is enough to show the existence and uniqueness of $r(q)$ : therefore showing this fact is the crucial step in the proof of Theorem 1.1.

First we show that, roughly speaking, the respective free boundaries are "almost" hyperplanes when the contact set is non-empty:

Lemma 3.2. (a) If $r<\bar{r}(q)-a$ and $q_{1}=(1+a) q$ with $a>0$ then

$$
d\left(\Gamma\left(\bar{u}_{\epsilon ; q_{1}, r}\right), l_{q_{1}, r}\right) \leq C \frac{\epsilon}{a} .
$$

(b) If $r>\underline{r}(q)+a$ and $q_{1}=(1-a) q$ with $a>0$, then

$$
d\left(\Gamma\left(\underline{u}_{\epsilon ; q_{1}, r}\right), l_{q_{1}, r}\right) \leq C \frac{\epsilon}{a} .
$$

By definition, $\bar{u}$ and $\underline{u}$ are harmonic functions in their support. Using local property of harmonic functions as well as above lemma, it is possible perturb $\bar{u}$ and $\underline{u}$ in above setting to construct local sub- and supersolutions whose free boundary, roughly speaking, curves away from the obstacle: one can perturb for example $\underline{u}$ this way when the obstacle speed is slower than $\underline{r}(q)$. We refer to the proof of Proposition 3.8 in [15] for details. One can then use these barriers and Theorem 2.1 to prove the following proposition:

Proposition 3.3. There exists a dimensional constant $C>0$ such that for any nonzero vector $q \in \mathbb{R}^{n}$ an for $r \neq 0$ the following is true:

(a) Let $r<\bar{r}(q)-a$ and $q_{1}=(1+a) q$ with $a>0$. Then for sufficiently small $\epsilon$ we have

$$
d\left(\Gamma\left(\underline{u}_{\epsilon ; q_{1}, r}\right), l_{q_{1}, r} \cap \tilde{Q}\right) \geq C \frac{\epsilon}{a} .
$$

for $t \geq \frac{C \epsilon}{|r| \gamma^{3}}$ 
(b) Let $r>\underline{r}(q)+a$ and $q_{1}=(1-a) q$ with $a>0$. Then

$$
d\left(\Gamma\left(\bar{u}_{\epsilon ; q_{1}, r}\right), l_{q_{1}, r} \cap \tilde{Q}\right) \geq C \frac{\epsilon}{a} .
$$

for $t \geq \frac{C \epsilon}{|r| \gamma^{3}}$

Above proposition (a) states that for obstacle speed slower than $\bar{r}(q)$, the free boundary of the minimal supersolution $\underline{u}$ above $P_{q, r}$ advances away from the obstacle. In particular it follows that $\bar{r}(q) \leq \underline{r}(q)$.

It remains then to show $\bar{r}(q) \geq \underline{r}(q)$. To show this, suppose $\bar{r}(q)<\underline{r}(q)$. Then for $\bar{r}(q)<r_{1}<$ $r_{2}<\underline{r}(q)$, both $\bar{u}_{q, r_{1}}$ and $\underline{u}_{q, r_{2}}$ are solutions of $(P)_{\epsilon}$ away from the parabolic boundary of $Q_{1}$ by definition. Now if one ignores what goes on near the boundary of $Q_{1}$, then translating $\underline{u}_{q, r_{2}}$ to put it below $\bar{u}_{q, r_{1}}$ at $t=0$ would yield a contradiction to Theorem 2.1 at a positive time $t=t_{0}>0$, as $\underline{u}$ crosses $\bar{u}$ from below. Finally to remove the boundary of $Q_{1}$ one can use a blow-up argument, using the fact that if one chooses $r_{1}$ and $r_{2}$ sufficiently close, respectively, to $\bar{r}(q)$ and $\underline{r}(q)$, the free boundaries of $\bar{u}$ and $\underline{u}$ are almost a hyperplane.

Thus we arrive at the following conclusion:

Lemma 3.4. For $q \in \mathbb{R}^{n}-\{0\}$, we have

$$
r(q):=\bar{r}(q)=\underline{r}(q) .
$$

In later sections of [14] it was shown, by proving Theorem 1.1, that $r(q)$ indeed is the correct limiting normal velocity. We mention that the perturbed test functions method introduced by Evans [10] has been applied to derive the result for solutions in general domains.

We mention that the limiting velocity $r(q)$ is nether independent of $q$ nor in the form of $|D u|-c\left(\frac{D u}{|D u|}\right)$, as one may expect from the form of the normal velocity in $(P)_{\epsilon}$. Indeed when $g(x)=g\left(x \cdot e_{1}\right)$, one can show (see [14]) that $r\left(a e_{1}\right)=0$ for $a \in[1,2]$ and, for unit vectors $\nu$ that are not $e_{1}, r\left(a_{0} \nu\right)=0$ for a unique constant $a_{0}$. Further properties of $r(q)$, in particular asymptotic behavior of $r(q)$ near "pinning" $q_{0}$ (i.e., $q_{0}$ such that $r\left(q_{0}\right)=0$ ) are of practical interest and is under investigation $([7])$.

Note that the statements of Proposition 3.3 are stronger than saying that the corresponding contact sets are empty. Indeed the proposition generates barriers with curved free boundaries, which is stable with respect to purturbations. For example it allows to compare obstacles facing different directions, yielding the continuity of $r(q)$ with respect to $q$.

Using the scaling $\tilde{u}^{\epsilon}(x, t):=\epsilon^{-1 / 2} u^{\epsilon}\left(\epsilon^{1 / 2} x, \epsilon^{1 / 2} t\right)$, translation by vectors in $\epsilon \mathbb{Z}^{n}$, and applying Proposition 3.3 with $\gamma=\frac{1}{\epsilon^{25}}$, the following quantitative version of above proposition has been derived in [15]:

Corollary 3.5 (Corollary 3.6, [15]). Let $C$ be the dimensional constant given in Proposition 3.3. Let $u^{\epsilon}$ solve $(P)_{\epsilon}$ in $\Sigma:=B_{4 \epsilon^{1 / 2}}(0) \times\left[-\alpha_{\epsilon}, 0\right]$, where

$$
\alpha_{\epsilon}:=\min \left[\frac{\epsilon^{4 / 5}}{|r|}, \epsilon^{3 / 5}\right] .
$$


(a) If $u^{\epsilon} \leq P_{q, r}$ in $\Sigma$ and if

$$
r_{0} \geq\left(1-\epsilon^{1 / 25}\right) r\left(\left(1+\epsilon^{1 / 25}\right) q\right)+2 \epsilon^{1 / 25}
$$

then

$$
d\left(\Gamma_{0}\left(\left(u^{\epsilon}\right)\right), l_{q, r}(0) \cap B_{\epsilon^{1 / 2}}(0)\right)>C \epsilon^{24 / 25} .
$$

(b) If $u^{\epsilon} \geq P_{q, r}$ in $\Sigma$ and if

$$
r_{0} \leq\left(1+\epsilon^{1 / 25}\right) r\left(\left(1-\epsilon^{1 / 25}\right) q\right)-2 \epsilon^{1 / 25},
$$

then

$$
d\left(\Gamma_{0}\left(u^{\epsilon}\right), l_{q, r}(0) \cap B_{\epsilon^{1 / 2}}(0)\right)>C \epsilon^{24 / 25} .
$$

\section{Error estimate}

Here we state the main error estimate result and sketch its proof.

Theorem 4.1 (Corollary 4.2, $[15])$. Suppose $u$ solves $(P)$ and $u^{\epsilon}$ solves $\left(P_{\epsilon}\right)$, with initial data $u_{0}$. Also suppose that $\Omega\left(u_{0}\right) \subset B_{R}(0)$ for some $R>0$ and that one of the conditions (I-a)-(I-c) holds. Then for any $T>0$, there exists positive constants $\epsilon_{0}=\epsilon\left(n, u_{0}, T\right)$ and $C_{0}=C(n, R)$ such that for $0<\epsilon, \epsilon_{0}$

$$
d\left((x, t), \Gamma\left(u^{\epsilon}\right)\right) \leq C_{0} \epsilon^{1 / 70} \text { for }(x, t) \in \Gamma(u) \cap[0, T] .
$$

The estimate (1) is perhaps far from being optimal: on the other hand, considering the highly nonlinear and nonlocal structure of the problem $\left(P_{\epsilon}\right)$, getting an optimal convergence rate seems to be a difficult question. See [4] and [6] for relevant discussions and results in the case of nonlinear elliptic PDEs in periodic and random media.

For simplicity, let us assume (I-a). To prove above theorem, we take the following perturbations of $u$. Define

$$
u_{1}(x, t):=u\left(\left(1+\epsilon^{1 / 70}\right)^{-1} x,\left(1+\epsilon^{1 / 70}\right)^{-1}\left(1-\epsilon^{1 / 60}\right) t\right)
$$

and the inf-convolutions

$$
v_{1}(x, t):=\inf _{|x-y| \leq \epsilon^{1 / 30}-\epsilon^{1 / 27} t} u_{1}(y, t),
$$

and

$$
w_{1}(x, t):=\inf _{|x-y|^{2}+|t-s|^{2} \leq \epsilon^{1 / 15}} v_{1}(y, s) .
$$

for $t \leq \epsilon^{-1 / 100} \leq \epsilon^{1 / 30-1 / 27}$.

Then due to the scaling law and Lemma $2.2, w_{1}$ is a viscosity supersolution of

$\left(P 1_{\epsilon}\right)$

$$
\begin{cases}-\Delta w_{1}=0 & \text { in }\left\{w_{1}>0\right\} \\ V=\left(1-\epsilon^{1 / 60}\right) r\left(\left(1+\epsilon^{1 / 70}\right) D w_{1}\right)+\epsilon^{1 / 27} & \text { on } \Gamma\left(w_{1}\right)\end{cases}
$$


Figure 1: Geometry at the contact point

Note that, as mentioned in section $1, w_{1}$ has exterior ball properties at its free boundary: the convolution has been performed twice to ensure that at each time $\Gamma_{t}\left(w_{1}\right)$ has both interior space ball and interior space-time balls.

Similarly, one can define $w_{2}$ by replacing infimum by supremum in (2)-(3). Then $w_{2}$ is a viscosity subsolution of

$$
\begin{cases}-\Delta w_{1}=0 & \text { in }\left\{w_{1}>0\right\} \\ V=\left(1+\epsilon^{1 / 60}\right) r\left(\left(1-\epsilon^{1 / 70}\right) D w_{1}\right)-\epsilon^{1 / 27} & \text { on } \Gamma\left(w_{1}\right)\end{cases}
$$

Note that, for $C$ depending on $R$ and $T \leq \epsilon^{-1 / 100}$,

$$
d\left(\Gamma_{t}\left(w_{k}\right), \Gamma_{t}(u)\right) \leq C \epsilon^{1 / 70} \text { for } 0 \leq t \leq T, k=1,2 .
$$

Due to (I-a), we have $w_{2} \leq u^{\epsilon} \leq w_{1}$ at $t=0$ and for $x \in K$. Thus, to prove Theorem 4.1 it suffices to show that this order is preserved over time: $w_{2} \leq u^{\epsilon} \leq w_{1}$ for $0 \leq t \leq T$.

Let us show that $u^{\epsilon} \leq w_{1}$. First suppose that $w_{1}$ is smooth, and assume that $\Gamma\left(u^{\epsilon}\right)$ touches $\Gamma\left(w_{1}\right)$ for the first time at $P_{0}=\left(x_{0}, t_{0}\right)$. Then $t_{0}>0$ and $u^{\epsilon} \leq w_{1}$ in $Q \cap\left\{t \leq t_{0}\right\}$. Let

$$
q_{0}=D w_{1}\left(P_{0}\right), \quad r_{0}=\frac{\left(w_{1}\right)_{t}}{\left|D w_{1}\right|}\left(P_{0}\right) .
$$

Note that, since $w_{1}$ is a supersolution of $\left(P 1_{\epsilon}\right)$, we have

$$
r_{0} \geq\left(1-\epsilon^{1 / 60}\right) r\left(\left(1+\epsilon^{1 / 70}\right) q_{0}\right)+\epsilon^{1 / 27} .
$$

If one can show that $u^{\epsilon}$ lies below an appropriate translation of $P_{q_{0}, r_{0}}$ whose free boundary touches $P_{0}$ in $B_{\epsilon^{1 / 2}}\left(x_{0}\right) \times\left[-\epsilon^{1 / 2}+t_{0}, t_{0}\right]$, then Theorem 4.1 yields a contradiction to the fact that $u^{\epsilon}$ touches $w_{1}$ from below at $P_{0}$ : hence we conclude that $u^{\epsilon} \leq w_{1}$. In the rest of the note I will explain how to apply to possibly non-smooth $w_{1}$ : for detailed arguments we refer to section 4 and 5 of [15].

Suppose $u^{\epsilon}$ crosses $w_{1}$ from below above at $t=t_{0}$, i.e., suppose

$$
0<t_{0}=\sup \left\{t: u^{\epsilon} \prec w_{1}\right\}<\epsilon^{-1 / 100}
$$

From the maximum principle for harmonic functions and from finite propagation properties of $\Gamma\left(u^{\epsilon}\right)$ and $\Gamma\left(w_{1}\right)$, one can verify that there exists $x_{0}$ such that

$$
\left\{u\left(\cdot, t_{0}\right)>0\right\} \subset\left\{w_{1}\left(\cdot, t_{0}\right)>0\right\} \text { and } \Gamma_{t_{0}}\left(u^{\epsilon}\right) \cap \Gamma_{t_{0}}\left(w_{1}\right)=\left\{x_{0}\right\}
$$

and $u^{\epsilon} \leq w_{1}$ for $t \leq t_{0}$.

Let us investigate the geometry of $\Gamma\left(w_{1}\right)$ at $P_{0}=\left(p_{0}, t_{0}\right)$. By definition of $w_{1}$, there is a space-time ball $B_{1}^{(n+1)}$ of radius $\epsilon^{1 / 30}$ with center $P_{1}=\left(p_{1}, t_{1}\right) \in \Gamma\left(v_{1}\right)$ touching $\Gamma\left(w_{1}\right)$ at $P_{0}$, On the other hand $u_{1}$ has an interior space ball of radius $B_{2}$ with center $p_{1}$ touching $\Gamma_{t_{1}}\left(u_{1}\right)$ at $p_{2}$ (see Figure 1 ). We rotate the coordinates such that 


$$
P_{0}-P_{1}=\left(d_{1} e_{1},-d_{2}\right) \in \mathbb{R}^{n} \times \mathbb{R}, \text { where } d_{1} \geq 0 \text { and } e_{1}=(1,0, \ldots, 0) .
$$

Observe that if $\Gamma\left(w_{1}\right)$ were smooth, $\frac{d_{2}}{d_{1}}$ equals the normal velocity of $\Gamma\left(w_{1}\right)$ at $P_{0}$. Let us briefly explain why $d_{1}$ is not zero: since $w_{1}$ has exterior space ball property and is harmonic in its positive set, one can verify by barrier argument that $\left|D w_{1}\right|$ is bounded on $\Gamma\left(w_{1}\right)$. By the motion law, it follows that $\Gamma\left(w_{1}\right)$ propagates with finite speed. This results in the fact that the interior space-time ball $B_{1}^{(n+1)}$ does not touch $P_{0}$ on its top or bottom, i.e. $d_{1} \neq 0$.

Now we define

$$
r_{0}=\frac{d_{2}}{d_{1}} \in[-2, \infty) \text { and } q_{0}=m e_{1},
$$

where

$$
m=\min _{x \in \Sigma} \frac{u_{1}\left(x+p_{2}, t_{1}\right)}{\epsilon^{1 / 10}}
$$

and $\Sigma=W \cap\left\{x \cdot e_{1}=\epsilon^{1 / 10}\right\}$, where

$$
W=\left\{x_{1}:=x \cdot e_{1} \geq 0,\left|x-x_{1} e_{1}\right| \leq\left(1-\epsilon^{1 / 70}\right)|x|\right\} .
$$

As mentioned above, $r_{0}$ is a natural candidate to replace $\frac{\left(w_{1}\right)_{t}}{\left|D w_{1}\right|}\left(P_{0}\right)$, and $e_{1}$ for the normal direction of $D w_{1}\left(\cdot, t_{0}\right)$ at $p_{0}$. The replacement for $\left|D w_{1}\right|\left(P_{0}\right), m$, is defined via $u_{1}$ : note that $(5)$ defines $m$ by the approximate gradient of $u_{1}$ in scale $\epsilon^{1 / 10}$ measured in a non-tangential cone $W$ with opening angle of $\epsilon^{1 / 70}$ away from the tangential hyperplane. Observe that

$$
\frac{w_{1}\left(p_{0}+\epsilon^{1 / 10} e_{1}, t_{0}\right)}{\epsilon^{1 / 10}} \leq m .
$$

Due to (6) and the regularity(exterior ball) property of $w_{1}$, it can be checked that $m$ yields an upper bound for $\left|D w_{1}\right|\left(x_{0}, t_{0}\right)$ with an error of order $O\left(\epsilon^{1 / 27}\right)$. Based on these observations and the monotonicity of $r(q)$ with respect to $|q|$, one concludes with (4).

Now it remains to verify the following statement:

$$
w_{1}(x, t) \leq P_{q_{0}, r_{0}}\left(\left(x+\epsilon^{29 / 30} e_{1}, t\right)+P_{0}\right) \text { in } B_{\epsilon^{1 / 2}}\left(x_{0}\right) \times\left(t_{0}-\alpha_{\epsilon}, t_{0}\right] .
$$

Indeed let $y_{0} \in \Sigma$ the point where $u_{1}\left(y_{0}, t_{1}\right)=m \epsilon^{1 / 10}$. By definition of $v_{1}$ we have

$$
v_{1}\left(x, t_{1}\right) \leq m \epsilon^{1 / 10} \text { in } D_{1}:=\left\{x:\left|x-y_{0}\right| \leq \epsilon^{1 / 30}-\epsilon^{1 / 27} t_{1}\right\} .
$$

Moreover, due to the definition the free boundary $\Gamma_{t_{1}}\left(v_{1}\right)$ touches an exterior space ball $\tilde{B}$ of radius $O\left(\epsilon^{1 / 30}\right)$ at $\left(p_{1}, t_{1}\right)$. This fact and (8) yields that $v_{1}\left(\cdot, t_{1}\right)$ is below the radial harmonic function $h$ in the ring domain

$$
\Pi:=\left(1+\epsilon^{1 / 15}\right) \tilde{B}-\tilde{B}
$$

with boundary data $h=m \epsilon^{1 / 10}$ in the outer part of $\partial \Pi$, and $h=0$ on the inner part. Since $h$ locally resembles $P_{q_{0}, r_{0}}$ near $\left(p_{1}, t_{1}\right)$, we have

$$
v_{1}(x, t) \leq P_{q_{0}+r_{0}}\left((x, t)+P_{0}\right)+O\left(\epsilon^{29 / 30}\right)
$$

in a small neighborhood of $\left(p_{1}, t_{1}\right)$. Lastly observe that, by definition, $v_{1}$ dominates the value of $w_{1}$ over space as well as time variables. This fact as well as (9) would yield the desired claim (7). 


\section{References}

[1] L. A. Caffarelli and A. Mellet, Capillary drops: contact angle hysteresis and sticking drops, Calc. Var. Partial Differential Equations 29 (2007), no. 2, 141-160.

[2] L. A. Caffarelli, K. A. Lee and A. Mellet, Singular limit and homogenization for flame propagation in periodic excitable media, Arch. Ration. Mech. Anal. 172 (2004), no. 2, 153190.

[3] L. A. Caffarelli, K. A. Lee and A. Mellet, Homogenization and flame propagation in periodic excitable media: the asymptotic speed of propagation, Comm. Pure Appl. Math. 59 (2006), no. $4,501-525$.

[4] L. A. Caffarelli and P. E. Souganidis, Rates of convergence for the homogenization of fully nonlinear uniformly elliptic pde in random media, Invent. Math. . 180 No. 2 (2010) pp. 301-360.

[5] L. A. Caffarelli, P. E. Souganidis, L. Wang, Homogenization of fully nonlinear, uniformly elliptic and parabolic partial differential equations in stationary ergodic media. Comm. Pure Appl. Math. 58 (2005), 319-361.

[6] F. Camilli, C. Marchi, Rates of convergence in periodic homogenization of fully nonlinear uniformly elliptic PDEs, Nonlinearity 22 (2009), pp. 1481-1498.

[7] S. Cho, A. Yip, I. C. Kim, in preparation

[8] M. G. Crandall and P. L. Lions, Viscosity solutions of Hamilton-Jacobi equations, Trans. Amer. Math. Soc. 277 , pp. 1-42.

[9] M. Crandall, H. Ishiii and P. L. Lions, Users' Guide to viscosity solutions of second order partial differential equations. Bull. Amer. Math. Soc. 27, No.1 (1992): 1-67.

[10] L. C. Evans, Periodic homogenisation of certain fully nonlinear partial differential equation, Proc. Roy. Soc. Edinburgh Sect. A. 120 (1992), 245-265.

[11] K. B. Glasner, Homogenization of contact line dynamics, Int. Free boundaries, 8 (2006), 523-542.

[12] K. B. Glasner and I. C. Kim, Viscosity solutions for a model of contact line motion, Interfaces Free Bound. 11 (2009), no. 1, 37-60.

[13] I. C. Kim, Homogenization of the free boundary velocity, Arch. Ration. Mech. Anal. 185 (2007), no. 1, 69-103.

[14] I. C. Kim, Homogenization of a model problem on contact angle dynamics, Comm. Partial Differential Equations 33 (2008), no. 7-9, 1235-1271.

[15] I. C. Kim, Error estimates on homogenization of free boundary velocities in periodic media, Ann. Inst. H. Poincar Anal. Non Linéaire 26 (2009), no. 3, 99-1019.

[16] I. C. Kim and A. Mellet, Homogenization of a Hele-Shaw problem in periodic and random media, Arch. Ration. Mech. Anal. 194 (2009), no. 2, 50-530.

[17] I. C. Kim and A. Mellet, Homogenization of one-phase Stefan-type problems in periodic and random media, Trans. Amer. Math. Soc. 362 (2010), no. 8, 4161-4190. 